\title{
Influence of Plasma Irradiation on Silkworm
}

\author{
Akira Yonesu, ${ }^{\mathrm{a},{ }^{*}}$ Kazunori Koga, ${ }^{\mathrm{b}}$ Masaharu Shiratani, ${ }^{\mathrm{b}}$ \& Nobuya Hayashic \\ aDepartment of Electrical and Electronics Engineering, University of the Ryukyus, Okinawa, \\ Japan; ' $F$ aculty of Information Science and Electrical Engineering, Kyushu University, Fukuoka, \\ Japan; 'Interdisciplinary Graduate School of Engineering Science, Kyushu University, Fukuoka, \\ Japan \\ *Address all correspondence to: Akira Yonesu, Department of Electrical and Electronics Engineering, University of \\ the Ryukyus, Nishihara-cho, Okinawa, Japan; Tel.:+81-98-8958692; Fax: +81-98-895-8708, E-mail: yonesu@eee.u- \\ ryukyu.ac.jp
}

\begin{abstract}
Silkworms have recently been proposed as an animal model for safety testing in basic research. We propose using silkworms for in vivo trials of direct plasma treatment. In this study, the influence of plasma irradiation on silkworms was investigated using a non-thermal atmospheric pressure plasma. Silkworm survival rate decreased with increasing low-frequency voltage and plasma irradiation period. Further investigation of the plasma-generated agents (oxygen related radicals, UV light, and charged particles), revealed that the contribution of charged particles significantly increases silkworm mortality.
\end{abstract}

KEY WORDS: silkworm survival rate, DBD plasma torch, non-thermal atmospheric pressure plasma, ozone, discharge current

\section{INTRODUCTION}

Non-equilibrium plasmas are characterized by the electrons in the plasma having a higher mean energy than the ions or atoms. This higher energy can produce sufficient active chemical radicals for the high-speed surface processing of materials without causing thermal damage. Recently, non-equilibrium atmospheric pressure plasma has been studied in direct contact with living tissues in order to deactivate pathogens, ${ }^{1}$ stop bleeding with no damage to healthy tissue, ${ }^{2}$ disinfect wounds and accelerate wound healing, ${ }^{3}$ and to selectively kill some types of cancer cells. ${ }^{4}$ However, much research is still required to clarify and minimize the health risks of plasma treatment. In particular, in vivo trials using animal models are essential for predicting the risks of plasma treatment. The use of mammals for experimental models has many associated problems, such as high cost and ethical issues. However, silkworms have been proposed recently as an animal model for safety testing in basic research, because the mechanisms of chemical absorption, distribution, metabolism, and excretion are similar in silkworms and mammals. ${ }^{5}$ Until now, silkworms have been used for assessing the therapeutic effects of chemicals and in toxicity research. ${ }^{6-11}$ Thus, silkworms are considered appropriate for evaluating the effects of plasma treatment on animal bodies. In this study, we made a preliminary investigation of the influence of plasma irradiation on live tissue using silkworms. 


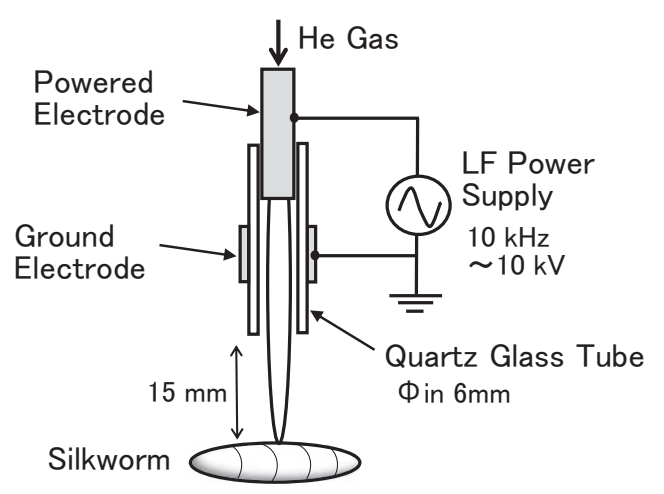

FIG. 1: Schematic diagram of the experimental setup

\section{EXPERIMENTAL APPARATUS AND METHOD}

Figure 1 shows the torch type of dielectric barrier discharge (DBD) plasma equipment used in this study. The quartz glass tube of 6-mm inner diameter was covered with copper tape as a ground electrode, and a copper tube inside the quartz glass tube functioned as the powered electrode. A DBD plasma jet was generated by applying low frequency (LF) high voltage $(10 \mathrm{kHz})$ to the two electrodes under atmospheric pressure. The working gas was helium. The LF voltage was varied from 6 to $9 \mathrm{kV}$, while the gas flow rate was fixed at 10 SLM in all cases. The length of the plasma plume increased from about $20 \mathrm{~mm}$ to $50 \mathrm{~mm}$ as the LF voltage was increased from $6 \mathrm{kV}$ to $9 \mathrm{kV}$. The life cycle of a silkworm consists of four stages: embryo, larva, pupa, and adult moth. The larval stage lasts for about 27 days, and the silkworm goes through five growth stages called instars during this time. Fifth instar silkworm larvae used in this study were purchased from Kogensha Co. Ltd (Nagano, Japan). The plasma irradiation was performed with a distance of $15 \mathrm{~mm}$ between the silkworm and the torch nozzle; the silkworm was in direct contact with the plasma. Therefore, the silkworms were exposed to all the plasma-generated agents (reactive species, UV photons, and charged particles). Ten silkworms were exposed individually to the plasma for each of the plasma treatment conditions. Ten untreated silkworms were used as a control. After plasma irradiation, both the treated and untreated silkworms were raised in a plastic case and fed artificial food twice a day. The amount of food administrated each time was $150 \mathrm{~g}$ per silkworm. The number of living silkworms was counted every day to obtain the survival rate after plasma irradiation.

The optical emission from the torch plasma was collected along the axial direction of the quartz tube using an optical fiber and was directed into a multi-channel spectrometer (Ocean Optics HR4000CG-UV-NIR). The ozone concentration at a distance of $20 \mathrm{~mm}$ from the torch nozzle, and the surface temperature of the silkworm, were measured using an ozone sensor (Eco Sensors, A-21ZX) and an infrared thermometer (Mather Tool, MT-10), respectively. The discharge voltage and current were measured 
with a high-voltage probe (Tektronix, P6015A) and current probe (Pearson, 4100), respectively, and monitored using a digital oscilloscope (Iwatsu, DS-5107).

\section{EXPERIMENTAL RESULTS AND DISCUSSION}

\section{A. Characteristics of Torch Plasma}

In order to evaluate species in the discharge region, we investigated the optical emission spectra with the LF voltage maintained at $7 \mathrm{kV}$. The typical optical emission spectrum is shown in Fig. 2, where the emission peaks of atomic oxygen $(\mathrm{O})$ and the hydroxyl radical $(\mathrm{OH})$ are observed. It is considered that these radicals were generated from the dissociation of oxygen molecules and water molecules in the ambient air. They are highly reactive and able to oxidize most chemicals. Moreover, significant UV emission spectra were not observed at wavelengths below $300 \mathrm{~nm}$. UV radiation in the 200 to $300 \mathrm{~nm}$ wavelength range with doses of several $\mathrm{mW} / \mathrm{sec} / \mathrm{cm}^{2}$ is known to cause lethal damage to cells. ${ }^{12}$ Thus, we expect the plasma-generated UV to not significantly affect the silkworms.

Another important reactive species generated in non-equilibrium atmospheric pressure plasma is ozone $\left(\mathrm{O}_{3}\right)$, because it is very long-lived at atmospheric pressure and so can be transported over long distances after being generated in the plasma. Figure 3 shows the $\mathrm{O}_{3}$ concentration at a distance of $20 \mathrm{~mm}$ from the torch nozzle as a function of LF voltage. It is found that the $\mathrm{O}_{3}$ concentration increases with increasing LF voltage up to a maximum concentration of $0.36 \mathrm{ppm}$ at a voltage of $9 \mathrm{kV}$. On the other hand, nitrogen-based reactive species, such as $\mathrm{NO}$ and $\mathrm{NO}_{2}$, were not detected by the gas detecting tubes (Kitagawa, 11L).

We evaluated the oxidation efficiency of oxygen-based reactive species generated in the torch plasma using chemical indicators (Sakura Color Products, Plazmark for $\mathrm{O}_{2}$ cleaning). The color of the chemical indicator changes gradually from purple to green by reaction with oxygen radicals, such that the oxidation efficiency can be evaluated. The plasma-irradiation-time dependence of the color of the chemical indicator with different LF voltages is shown in Fig. 4. The color of the chemical indicators was found to change

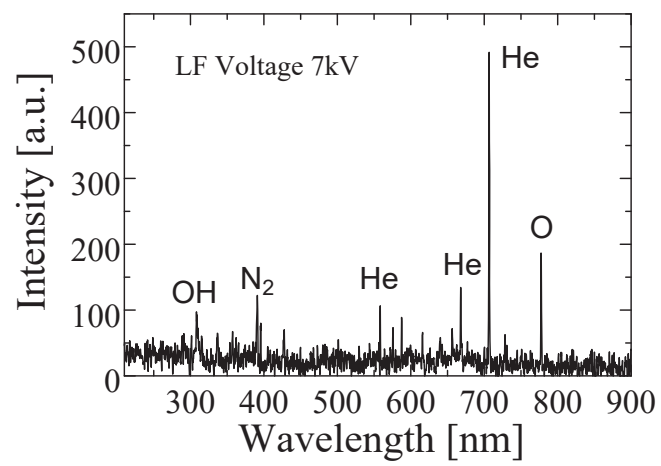

FIG. 2: Optical emission spectra. LF voltage: $7 \mathrm{kV}$

Volume 7, Issue 4, 2017 


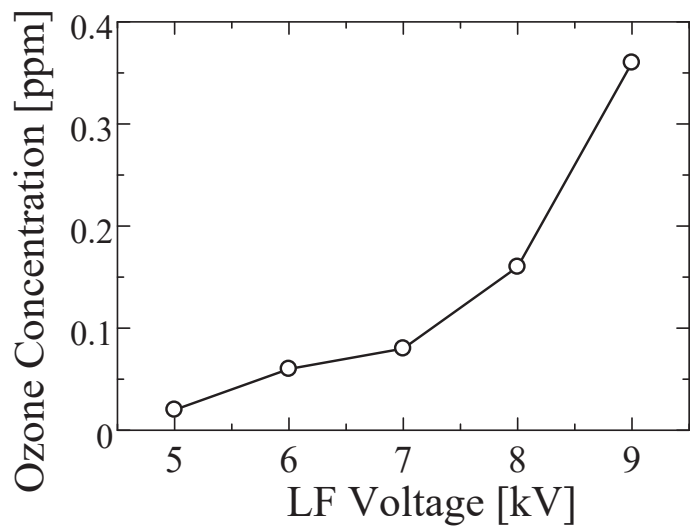

FIG. 3: Ozone concentration as a function of LF voltage

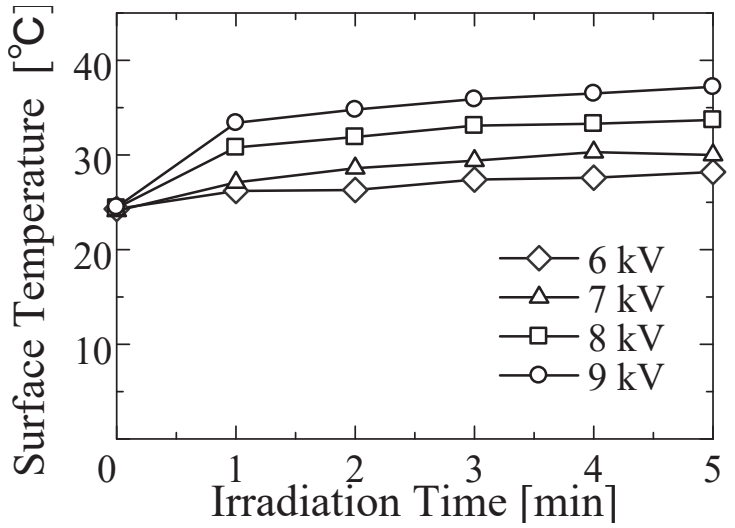

FIG. 4: Plasma irradiation time dependence of the color of the chemical indicator with different LF voltages

with the plasma irradiation, meaning that oxygen radicals with the ability to undergo surface oxidation exist at the plasma irradiation point.

The surface temperatures of the silkworm at the plasma irradiation point as a function of plasma irradiation time for different LF voltage are shown in Fig. 5. Under our plasma irradiation conditions, the surface temperatures of the silkworm were lower than about $38^{\circ} \mathrm{C}$. The viable temperature of silkworms is $7^{\circ} \mathrm{C}$ to $40^{\circ} \mathrm{C}$, so plasma irradiation is unlikely to cause thermal damage to silkworms.

\section{B. Plasma Irradiation of Silkworms}

Figure 6 shows the time variation of the survival rate of silkworms after plasma irradiation in several LF voltages with a fixed plasma irradiation period of $2 \mathrm{~min}$. The control silkworms that were not irradiated by plasma exhibited $100 \%$ survival. No color 
changes were observed on the surface of the silkworm immediately after plasma irradiation in all conditions. However, the color of the silkworm surface changes to black about three hours after the plasma irradiation. It is demonstrated that the survival rate of irradiated silkworms begins to decrease more rapidly in cases of higher LF voltage, as shown in Fig. 6. Figure 7(a) shows the survival rate of the silkworm 10 days after plasma irradiation for $2 \mathrm{~min}$ as a function of the LF voltage. It is found that the survival rate decreased proportionally with increasing LF voltage. In addition, the survival rate of the silkworms 10 days after plasma irradiation as a function of the plasma irradiation period for an LF voltage of $7 \mathrm{kV}$ is shown in Fig. 7(b). It is found that the survival rate decreased proportionally with increasing plasma irradiation period.

To evaluate only the effect of ozone on the silkworm, we performed a further $\mathrm{O}_{3}$ treatment experiment. Ten silkworms were placed in a sealed plastic container $(160 \times$ $100 \times 30 \mathrm{~mm}$ ), and ozone gas was introduced. Ozone was generated by a self-designed ozonizer that utilized surface discharge. The working gas was $\mathrm{O}_{2}$, and $\mathrm{O}_{3}$ concentration in the container was controlled by varying the LF voltage. Ozone concentration was measured using a gas detecting tube (Kiragawa, 17S). Figure 8 shows the survival rate of silkworms as a function of $\mathrm{O}_{3}$ dose $\left(\mathrm{O}_{3}\right.$ concentration multiplied by irradiation period). It is found that the threshold $\mathrm{O}_{3}$ dose for death of the silkworm is about 5,000 ppm-min. This value is much larger than that of the previously mentioned plasma irradiation using the plasma torch. Thus, it seems that the contribution of $\mathrm{O}_{3}$ to silkworm

\begin{tabular}{|c|c|c|c|c|}
\hline & \multicolumn{4}{|c|}{ Treatment time [min] } \\
\hline Voltage [kV] & 0 & 1 & 3 & 5 \\
\hline 6 & & & & \\
\hline 8 & & & & \\
\hline
\end{tabular}

FIG. 5: Surface temperature of silkworms as a function of plasma irradiation time for different LF voltages

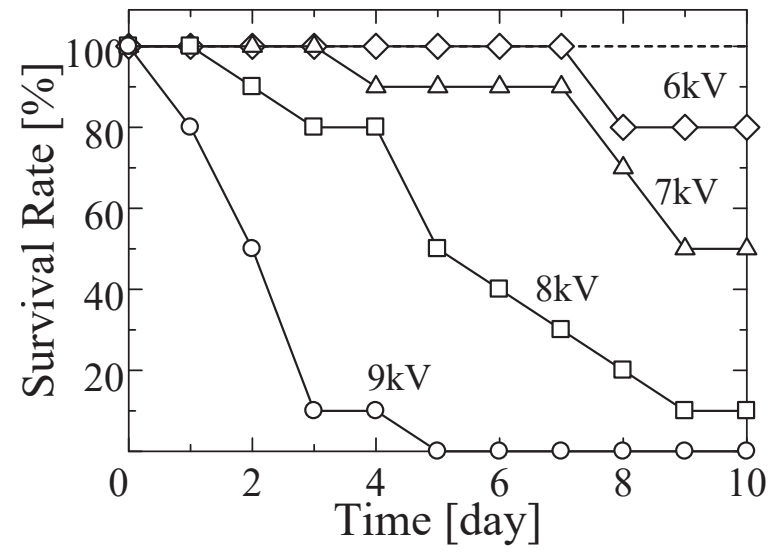

FIG. 6: Time variation of survival rate of silkworm with several LF voltages 

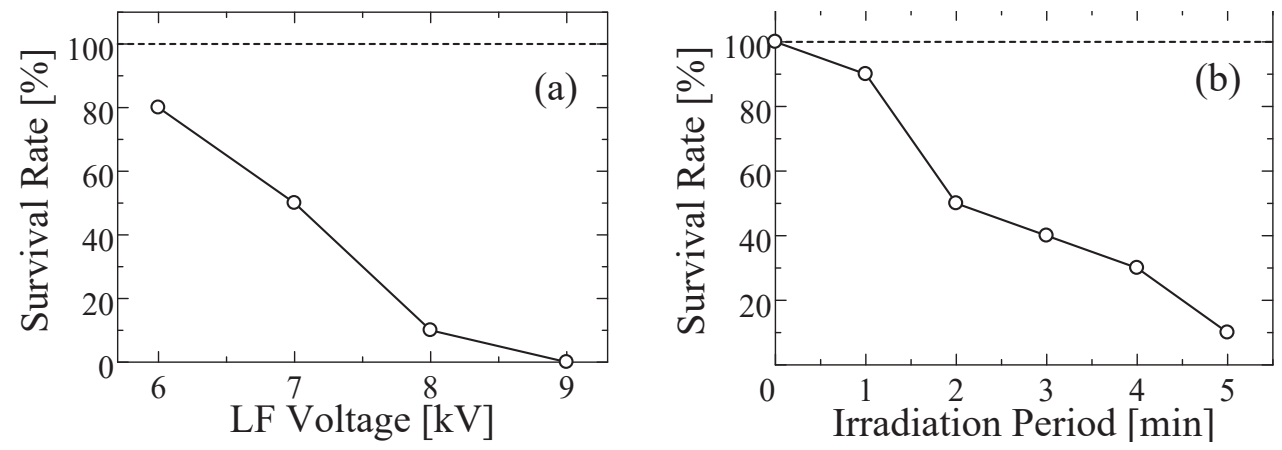

FIG. 7: Survival rate of silkworms 10 days after plasma irradiation for 2 min as a function of LF voltage (a), and as a function of plasma irradiation period for a voltage of $7 \mathrm{kV}$ (b)

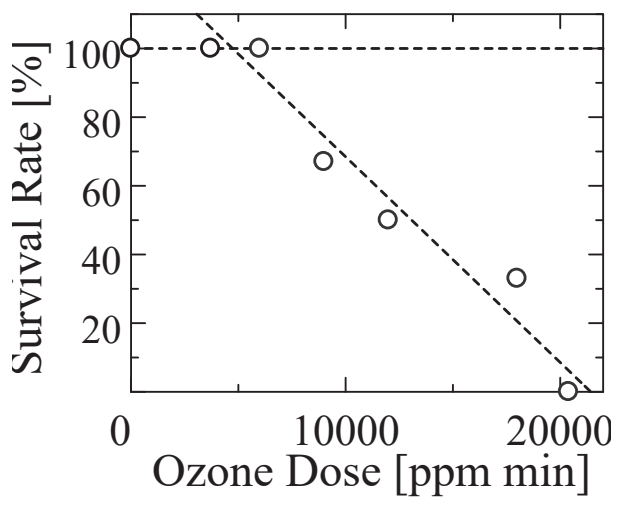

FIG. 8: Survival rate of silkworms as a function of the ozone dose (ozone concentration multiplied by irradiation period)

mortality in torch plasma irradiation is negligible.

Figure 9 shows the root-mean-square (RMS) of discharge currents and the optical emission intensity ratio between oxygen atoms $(777 \mathrm{~nm})$ and helium atoms $(706 \mathrm{~nm})$ as a function of LF voltage. These optical emission intensity ratios can be approximately regarded as the existence ratio between oxygen atoms and helium atoms in the plasma. ${ }^{13}$ The optical emission ratio shown in Fig. 9 indicates the variation of the amount of oxygen atoms, since the helium gas flow rate was fixed in this experiment. It is found that the discharge current (which is the flux of charged particles) is proportional to the LF voltage, whereas the optical emission intensity ratio (namely, the amount of oxygen atoms) is almost independent of the LF voltage. As mentioned before, the survival rate of silkworms decreases with increasing LF voltage (Fig. 7[a]). Therefore, we conclude that the survival rate of the silkworm is inversely correlated with the presence of charged 


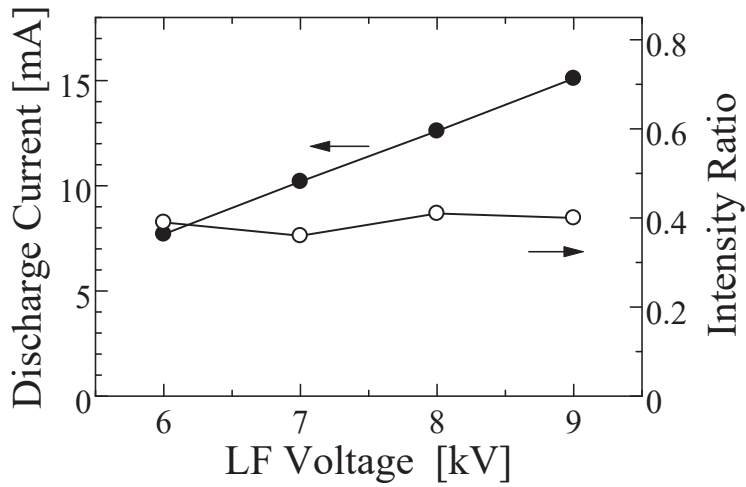

FIG. 9: RMS of discharge currents and optical emission intensity ratio between oxygen atoms $(777 \mathrm{~nm})$ and helium atoms (706 nm) as a function of LF voltage

particles. Consequently, we propose that the contribution of charged particles is a significant cause of silkworm mortality. Regarding the role of the charged particles, it has been reported that when direct non-thermal plasma treatment is employed, the charged particles play an important role in the inactivation of bacteria. ${ }^{14-16}$

\section{CONCLUSION}

We investigated the influence of plasma irradiation on silkworms using a non-thermal plasma torch. The survival rate of the silkworm decreases proportionally with increasing LF voltage and plasma irradiation period. It was found that the contribution of charged particles significantly affects silkworm mortality. In this study, we have investigated the survival rate of silkworms when exposed to plasma. Further studies are still needed to better understand the mechanism of silkworm mortality by plasma irradiation.

\section{ACKNOWLEDGMENT}

This work was supported by JSPS KAKENHI Grant Number 24108009.

\section{REFERENCES}

1. Scholtz V, Pazlarova J, Souskova H, Khun J, Julak J. Nonthermal plasma-a tool for decontamination and disinfection. Biotechnol Adv. 2015;33:1108-19.

2. Fridman G, Peddinghaus M, Ayan H, Fridman A, Balasubramanian M, Gutsol A, Brooks A, Friedman G. Blood coagulation and living tissue sterilization by floating-electrode dielectric barrier discharge in air. Plasma Chem Plasma Process. 2006;26:425-42.

3. Isbary G, Morfill G, Schmidt HU, Georgi M, Ramrath K, Heinlin J, Karrer S, Landthaler M, Shimizu T, Steffes B, Bunk W, Monetti R, Zimmerman JL, Pompl R, Stolz W. A first prospective randomized controlled trial to decrease bacterial load using cold atmospheric argon plasma on chronic wounds in patients. Br J Dermatol. 2010;163(1):78-82.

Volume 7, Issue 4, 2017 
4. Fridman G, Shereshevsky A, Jost M, Brooks A, Friedman A, Gutsol A, Vasilers V, Friedman G. Floating electrode dielectric barrier discharge plasma in air promoting apoptotic behavior in melanoma skin cancer cell lines. Plasma Chem Plasma Process. 2007;27:163-76.

5. Hamamoto H, Tonoike A, Narushima K, Hori R, Sekimizu K. Silkworm as a model animal to evaluate drug candidate toxicity and metabolism. Comp Biochem Physiol C Toxicol Pharmacol. 2009;149:3349 .

6. Ishii K, Hamamoto H, Kamimura M, Sekimizu K. Activation of the silkworm cytokine by bacterial and fungal cell wall components via a reactive oxygen species-triggered mechanism. J Biol Chem. 2008;283:2185-91.

7. Matumoto Y, Sumiya E, Sugita T, Sekimizu K. An invertebrate hyperglycemic model for identification of anti-diabetic drugs. PLoS One. 2011;6(3):18292.

8. Fjiyuki T, Imamura K, Hamamoto H, Sekimizu K. Evaluation of therapeutic effects and pharmacokinetics of antibacterial chromogenic agents in a silkworm model of staphylococcus aureus infection. Drug Discov Ther. 2010;4:349-54.

9. Hamamot H, Sekimizu K. Evaluation of therapeutic effects of antibiotics using silkworm as an animal model. Res Adv Antimicrob Agents Chemother. 2005;5:1-23.

10. Kaito C, Akimitsu N, Watanabe H, Sekimizu K. Silkworm larvae as an animal model of bacterial infection pathogenic to humans. Microb Pathog. 2002;32:183-90.

11. Hamamoto H, Kurokawa K, Kaito C, Kamura K, Mantitra Razanajatovo I, Kusuhara H, Santa T, Sekimizu K. Quantitative evaluation of the therapeutic effects of antibiotics using silkworms infected with human pathogenic microorganisms. Antimicrob Agents Chemother. 2004;48:774-9.

12. Munakata N, Sato M, Hieda K. Inactivation action spectra of Bacillus subtilis spores in extended ultraviolet wavelength obtained with synchrotron radiation. Photochem Photobiol. 1991;54:761-8.

13. Coburn JW, Chen M. Optical emission spectroscopy of reactive plasmas: a method for correlating emission intensities to reactive particle density. J Appl Phys. 1980;51:3134-6.

14. Fridman G, Brooks A, Balasubramanian M, Fridman A, Gutsol A, Vasilets NV, Ayan H, Friedman G. Comparison of direct and indirect effects of non-thermal atmospheric-pressure plasma on bacteria. Plasma Process Polym. 2007;4:370-5.

15. XinPei L, Tao Y, YingGuang C, ZiYong S, Qing X. ZhiYuan T, ZhiLan X, Jing H, ZhongHe J, Yuan P. The roles of the various plasma agents in the inactivation of bacteria. J Appl Phys. 2008 Sep 15;104(053309):1-5.

16. Dobrymin D, Fridman G, Friedman G, Fridman A. Physical and biological mechanisms of direct plasma interaction with living tissue. New J Phys. 2009;11(115020):1-26. 